\title{
Pharmacovigilance Evaluation of Bendamustine-related Skin Disorders using the Japanese Adverse Drug Event Report Database
}

\author{
Mayako Uchida $^{1}$, Takehiro Kawashiri ${ }^{2}$, Nami Maegawa ${ }^{1}$, Aoi Takano ${ }^{1}$, Keiko Hosohata ${ }^{1}$, Yoshihiro Uesawa ${ }^{3}$ \\ ${ }^{1}$ Education and Research Center for Clinical Pharmacy, Osaka University of Pharmaceutical Sciences, Takatsuki, \\ Japan; ${ }^{2}$ Department of Clinical Pharmacy and Pharmaceutical Care, Graduate School of Pharmaceutical Sciences, \\ Kyushu University, Maidashi, Higashi-ku, Fukuoka, Japan; ${ }^{3}$ Department of Medical Molecular Informatics, Meiji \\ Pharmaceutical University, Kiyose, Japan
}

Corresponding author: Mayako Uchida, Ph.D., Education and Research Center for Clinical Pharmacy, Osaka University of Pharmaceutical Sciences, 4-20-1 Nasahara, Takatsuki, Osaka 569-1094, Japan; TEL: (+81)-72-690-1273; Fax: (+81)72-690-1023; mayaco@gly.oups.ac.jp

Received, November 16, 2020; Revised, January 4, 2021; Accepted, January 4, 2021; Published, January 7, 2021

\begin{abstract}
Purpose: Bendamustine is used in hematologic malignancies such as non-Hodgkin lymphoma, chronic lymphocytic leukemia, and multiple myeloma. This study evaluated the association of bendamustinerelated skin disorders using the Japanese Adverse Drug Event Report (JADER) database. Methods: We identified and analyzed reports of skin disorders between April 2004 and November 2019 from the JADER database and calculated the reported odds ratios (RORs) using disproportionality analysis. Additionally, we analyzed the relationship between skin disorders related to bendamustine use and patient information (age and sex). Results: The symptoms, ranked in order of decreasing strength of association with skin disorders, were infusion-related reaction $(\mathrm{ROR}=5.708)$, herpes zoster $(\mathrm{ROR}=4.658)$, hypersensitivity $(\mathrm{ROR}=3.271)$, and rash $(\mathrm{ROR}=1.472)$. Additionally, analysis of the relationships between rash related to bendamustine and sex or age showed significant relationships for female sex and age younger than 70 years ( $R O R=2.247$ and 2.176, respectively). Meanwhile, analysis of the relationship between herpes zoster and sex showed a significantly stronger association for male than female sex $(\mathrm{ROR}=2.887)$. Conclusion: Our analysis of skin disorders related to bendamustine use reported in the spontaneous reporting system databases showed that the association of rash with bendamustine use was affected by sex (female) and age (younger than 70 years). Additionally, the association of herpes zoster with bendamustine was affected by sex (male). Bendamustine is an outpatient chemotherapy regimen, and so we recommend close monitoring of female patients or those younger than 70 years who experience rash-like symptoms and male patients who experience herpes zoster-like symptoms.
\end{abstract}

\section{INTRODUCTION}

Bendamustine is widely used as first-line therapy for patients with non-Hodgkin lymphoma (NHL) (1) and chronic lymphocytic leukemia (CLL) $(1,2)$. Additionally, bendamustine is highly effective in patients with relapsed/refractory or elderly patients with multiple myeloma (MM) $(3,4)$. Therefore, bendamustine therapy is being increasingly used in various hematologic malignancies such as NHL, CLL, and MM.

However, bendamustine can cause various skin disorders in patients. A phase II study in the United States reported skin disorders in $9.2 \%$ of patients with NHL receiving bendamustine alone (5). Other reports in the Western world indicate a skin disorder frequency of $5.0-9.3 \%$ for bendamustine monotherapy in patients with CLL (2). Meanwhile, a phase II study in Japan reported skin disorders in
$46.4 \%$ of patients with NHL treated with bendamustine alone (6). This evidence suggests that bendamustine-related skin disorders occur at higher frequencies in Japan. These skin disorders can have profound impacts on patient health-related quality of life through both physical discomfort and psychological distress (7-9). Moreover, worsening skin symptoms may lead to dose reduction/termination in the management of cancer chemotherapy $(7,8)$. Therefore, it is important to clarify the risk factors for skin toxicities after bendamustine treatment.

Recently, spontaneous reporting systems have been used as essential methods of post-marketing drug safety surveillance to detect adverse drug events (ADEs) $(10,11)$. While randomized clinical trials are considered the gold standard for assessing drug efficacy and safety $(10,12)$, the design of such trials involves small and homogeneous populations 
that are monitored over short periods, making it difficult to detect many ADEs (13). Thus, the detection and reporting of suspected ADEs in clinical practice is the backbone of post-marketing surveillance (14). Therefore, pharmacovigilance activities are important for assessing, monitoring, and preventing ADEs (11). In April 2004, Japan's Pharmaceuticals and Medical Devices Agency (PMDA) established a spontaneous reporting system. The Japanese Adverse Drug Event Report (JADER) database is a large-scale, unitized database for pharmacovigilance that accumulates reported cases and their results (15-17). To our knowledge, the present study is the first report to analyze the relevant characteristics of skin disorders in bendamustinetreated patients using a database of adverse events reported spontaneously in Japan. This study evaluated the association of bendamustine-related skin disorders using the Japanese Adverse Drug Event Report (JADER) database. Patient background (e.g., age and sex) is an important factor to consider when monitoring ADEs in the context of pharmacovigilance. In a phase II study in Japan of bendamustine plus rituximab, the incidence of CD4 lymphopenia was 93\%. Patients with CD4 lymphocytopenia are susceptible to various opportunistic infections such as herpes zoster (18). Herpes zoster is a rash that most often appears as a band of rashes or blisters in one area of the body; it gradually becomes more painful, sometimes enough to disrupt sleep.

ABBREVIATION. ADEs: adverse drug events; CLL: chronic lymphocytic leukemia; DEMO: demographic information; DRUG: drug information; HIST: medical history; IL-2: interleukin-2; JADER: Japanese adverse drug event report; NHL: non-Hodgkin lymphoma; MedDRA: medical dictionary for regulatory activities; MM: multiple myeloma; PMDA: pharmaceuticals and medical devices agency; PT: preferred term; REAC: adverse events; RORs: reported odds ratios; SNPs: single nucleotide polymorphisms; 95\% CIs: $95 \%$ confidence intervals

\section{MATERIALS AND METHODS}

Data from released JADER database (15-17). This database is available for free download from the PMDA website (http://www.pmda.go.jp) and includes ADE cases. We analyzed ADE reports recorded between April 2004 and November 2019. The data structure of the JADER consists of four datasets: patient demographic information (DEMO), drug information (DRUG), adverse events (REAC), and medical history (HIST). The REAC table uses the Medical Dictionary for Regulatory Activities (MedDRA) to codify the ADEs, which are indicated as "Preferred Term (PT)"
We first removed duplicate cases from the DRUG and REAC tables, as described by Hirooka and Yamada (19). We then used the identification number of each ADEs case to merge corresponding case data from the DRUG, REAC, and DEMO tables. The medication contributions to the ADEs were classified as "suspected medicine," "concomitant medicine," and "interaction." We only extracted cases that were classified as "suspected medicine," which included 1,584,551 cases. Additionally, cases with incomplete information on patient age and sex were removed (114,814 cases). We used more than five reports for each skin disorder.

We evaluated the association of skin disorders with bendamustine based on the reported odds ratios (RORs). ROR is frequently used in the spontaneous reporting database as an indicator of the relative risk of ADEs. We used the analysis data table and constructed $2 \times 2$ tables based on two classifications: the presence or absence of the "skin disorders" and the presence or absence of suspected bendamustine use. We next calculated the ROR as the reporting rate of a "skin disorder" caused by bendamustine divided by the rate of the same adverse event caused by all other drugs present in the database. Skin disorder signals were considered positive when the lower limits of the $95 \%$ confidence intervals (95\% CIs) of the ROR were $>1$. Additionally, we analyzed the relationships between bendamustine-related skin disorders and patient information (sex and age). The objective and explanatory variables were set to skin disorders and patient characteristics (age and sex), respectively. We calculated the RORs, 95\% CIs, and $p$-values using Fisher's exact tests. Ages reported in the JADER database are provided as delimited data every 10 years owing to privacy considerations. Although elderly people are generally defined as those aged 65 years or older, some researchers have proposed increasing this threshold to reflect the longevity and aging of the Japanese population (20). Therefore, we categorized patients as 70 years and older and under 70 years. All data were analyzed using JMP 14.2.0 (SAS Institute Inc., Cary, NC, U.S.A.), with $\mathrm{p}<0.05$ indicating statistical significance.

\section{RESULTS}

Among 1,469,737 cases, we identified 1,967 reports in which patients were administered bendamustine between April 2004 and November 2019, including 136 reports on bendamustine-related skin disorders. Of the 136 reports, 35 cases $(25.73 \%)$ were combined with rituximab (Table 1). 
Table 1. Numbers of reports and RORs of skin disorders related to bendamustine

\begin{tabular}{|c|c|c|c|c|c|c|c|}
\hline Variable & Cases (n) & Non-cases (n) & $\begin{array}{l}\text { Rate } \\
(\%)\end{array}$ & $\begin{array}{c}\text { Cases of combination } \\
\text { with rituximab (n) }\end{array}$ & ROR & $95 \% \mathrm{CI}$ & $p$ \\
\hline Rash & 32 & 1935 & 1.627 & 10 & 1.472 & $1.037-2.088$ & 0.039 \\
\hline Herpes zoster & 24 & 1943 & 1.220 & 3 & 4.658 & $3.111-6.975$ & $<0.0001$ \\
\hline Stevens-Johnson syndrome & 18 & 1949 & 0.915 & 6 & 1.243 & $0.781-1.977$ & 0.354 \\
\hline Infusion-related reaction & 14 & 1953 & 0.712 & 4 & 5.708 & $3.368-9.675$ & $<0.0001$ \\
\hline Drug eruption & 11 & 1956 & 0.559 & 3 & 0.574 & $0.317-1.038$ & 0.065 \\
\hline Erythema multiforme & 8 & 1959 & 0.407 & 2 & 0.648 & $0.323-1.298$ & 0.253 \\
\hline Erythema & 8 & 1959 & 0.407 & 3 & 0.988 & $0.493-1.980$ & 1.000 \\
\hline Hypersensitivity & 8 & 1959 & 0.407 & 2 & 3.271 & $1.631-6.560$ & 0.004 \\
\hline Toxic epidermal necrolysis & 8 & 1959 & 0.407 & 2 & 0.812 & $0.405-1.627$ & 0.747 \\
\hline Toxic skin eruption & 5 & 1962 & 0.254 & 0 & 1.216 & $0.505-2.927$ & 0.615 \\
\hline
\end{tabular}

Cases indicates the number of cases reporting with skin disorders. ROR: reporting odds ratio. 95\% CI: 95\% confidence interval. Italicized $p$-values represent statistically significant results. We used more than 5 reports for each skin disorder. 
The symptoms ranked in order of descending number of reports of skin disorders were rash (PT code 10037844, 32 reports), herpes zoster (PT code 10019974, 24 reports), stevens-Johnson syndrome (PT code 10042033, 18 reports), infusion-related reaction (PT code 10051792, 14 reports), drug eruption (PT code 10013687, 11 reports), erythema multiforme (PT code 10015218, 8 reports), erythema (PT code 10015151, 8 reports), hypersensitivity (PT code 10020751, 8 reports), toxic epidermal necrolysis (PT code 10044223, 8 reports), and toxic skin eruption (PT code 10057970, 5 reports). The ROR does not allow for the quantification of risk factors but, rather, indicates an increased risk of ADEs reporting. Signals were detected in four of 10 ADEs for skin disorders. The symptoms, ranked in order of decreasing strength of association with skin disorders, were infusion-related reactions 5.708 (3.368 to 9.675), herpes zoster 4.658 (3.111 to
6.975), hypersensitivity 3.271 (1.631 to 6.560$)$, and rash 1.472 (1.037 to 2.088 ).

Tables 2 and 3 show the RORs for age and sex for each skin and non-skin disorder of the 10 skin disorders listed in Table 1. This study population comprised 1,967 cases of bendamustine-associated ADEs, including 136 skin disorders. Of the adverse event reports associated with the administration of bendamustine, the rate of reported skin rashes was significantly higher in female patients than in male patients (ROR, 2.247; 95\% CI, 1.092-4.623; $\mathrm{p}=0.030$ ) and in younger patients than in elderly patients (ROR, 2.176; 95\% CI, 1.002-4.728; $\mathrm{p}=0.049$ ). Among the adverse event reports associated with bendamustine, the rate of herpes zoster in male patients was higher than that in female patients (ROR, 2.887; 95\% CI, 1.074-7.766; $\mathrm{p}=0.036)$.

Table 2. ROR of skin disorders related to sex using adverse event reports for bendamustine

\begin{tabular}{lccrrr}
\hline \multirow{2}{*}{ Variable } & \multirow{2}{*}{ ROR } & $\mathbf{9 5 \%}$ CI & & \multicolumn{2}{c}{ Numbers of skin disorders reports } \\
\cline { 5 - 6 } & & & $\boldsymbol{p}$ & Women & Men \\
\hline Rash & 2.247 & $1.092-4.623$ & 0.030 & 20 & 12 \\
Herpes zoster & 0.346 & $0.129-0.931$ & 0.036 & 5 & 19 \\
Stevens-Johnson syndrome & 1.334 & $0.527-3.376$ & 0.635 & 9 & 9 \\
Infusion-related reaction & 0.738 & $0.246-2.209$ & 0.788 & 5 & 9 \\
Drug eruption & 0.759 & $0.222-2.602$ & 0.767 & 4 & 4 \\
Erythema multiforme & 1.332 & $0.332-5.342$ & 0.731 & 4 & 5 \\
Erythema & 0.798 & $0.190-3.347$ & 1.000 & 3 & 7 \\
Hypersensitivity & 0.189 & $0.023-1.540$ & 0.149 & 1 & 5 \\
Toxic epidermal necrolysis & 0.798 & $0.190-3.347$ & 1.000 & 3 & 2 \\
Toxic skin eruption & 1.999 & $0.333-11.992$ & 0.657 & 3 & 5 \\
\hline
\end{tabular}

Table 3. ROR of skin disorders related to age using adverse event reports for bendamustine

\begin{tabular}{|c|c|c|c|c|c|}
\hline \multirow[b]{2}{*}{ Variable } & \multirow[b]{2}{*}{ ROR } & \multirow[b]{2}{*}{$95 \%$ CI } & \multirow[b]{2}{*}{$p$} & \multicolumn{2}{|c|}{ Numbers of skin disorders reports } \\
\hline & & & & $\begin{array}{l}\text { Elderly } \\
(\geqq 70)\end{array}$ & $\begin{array}{l}\text { Young } \\
(<70)\end{array}$ \\
\hline Rash & 0.459 & $0.212-0.998$ & 0.049 & 9 & 23 \\
\hline Herpes zoster & 1.191 & $0.532-2.663$ & 0.686 & 12 & 12 \\
\hline Stevens-Johnson syndrome & 0.950 & $0.373-2.417$ & 1.000 & 8 & 10 \\
\hline Infusion-related reaction & 0.322 & $0.089-1.157$ & 0.104 & 3 & 11 \\
\hline Drug eruption & 0.990 & $0.301-3.254$ & 1.000 & 5 & 6 \\
\hline Erythema multiforme & 0.712 & $0.170-2.987$ & 0.734 & 3 & 5 \\
\hline Erythema & 0.712 & $0.170-2.987$ & 0.734 & 3 & 5 \\
\hline Hypersensitivity & 1.189 & $0.296-4.767$ & 1.000 & 4 & 4 \\
\hline Toxic epidermal necrolysis & 0.712 & $0.170-2.987$ & 0.734 & 3 & 5 \\
\hline Toxic skin eruption & undetected & undetected & undetected & 0 & 5 \\
\hline
\end{tabular}




\section{DISCUSSION}

The JADER database collects comprehensive information on ADEs from real-world data. Therefore, this pharmacovigilance study was suitable for the evaluation of ADE signals in various skin disorders related to bendamustine.

Our study results showed that skin disorders such as infusion-related reaction, herpes zoster, hypersensitivity, and rash were associated with bendamustine. Structurally, bendamustine consists of three parts; namely, a mechlorethamine group with alkylating properties, a butyric acid side chain that enhances water solubility, and a benzimidazole ring that confers an antimetabolite property. $(21,22)$ A review of current literature reported a relationship between aromatic drugs and increased risks of rash or hypersensitivity reactions. $(23,24)$ Additionally, Knowles et al reported that chemical structures containing aromatic rings may be involved in the mechanism that contributes to skin disorders. (25) Our results showed that infusion-related reaction, hypersensitivity, and rash were signals of adverse events specific to bendamustine. Thus, skin damage caused by the use of bendamustine may be an allergic reaction to its aromatic ring.

The results of the present study also indicated that herpes zoster was an adverse event signal specific to bendamustine use. In a phase II study in Japan of bendamustine plus rituximab, the incidence of CD4 lymphopenia was $93 \%$ (26). Patients with CD4 lymphocytopenia are susceptible to various opportunistic infections such as herpes zoster (27). Moreover, the results of a phase II study of NHL patients showed that a frequency of opportunistic infection such as herpes zoster of $4.3 \%$ after the initiation of bendamustine alone therapy (6). Herpes zoster due to bendamustine may be caused by immunosuppression. It is unclear whether the patients who experienced herpes zoster in our study were prophylactically treated with antiviral medications. Although there is no mention in the National Comprehensive Cancer Network guidelines of prophylactic administration of antiviral medications when administering bendamustine, this prophylactic administration should be considered for recommendation.

Additionally, we demonstrated that female sex and age younger than 70 years were important risk factors for rash, whereas male sex was an important risk factor for herpes zoster in the JADER database. The potential impacts of sex and age on the pharmacokinetics of bendamustine have been evaluated in both adult and pediatric patients but are not considered to be affected by either factor (22).
The details of the sex- and age-based mechanisms underlying rash and herpes zoster associated with bendamustine use are not fully understood. However, there is a difference in the occurrence of skin disorders between those with and without mutations in single nucleotide polymorphisms (SNPs) that suppress Interleukin-2 (IL-2) expression,

suggesting that SNPs are associated with the occurrence of bendamustine-related skin disorders (28). IL2 plays an important role in the T-cellmediated immune response and exerts antitumor activity (28). Additional

studies are needed to evaluate these relationships between SNPs mutations and sex and age.

In conclusion, our results showed that infusion-related reaction, herpes zoster, hypersensitivity, and rash were bendamustinerelated skin disorders. Additionally, we demonstrated that female sex and age younger than 70 years were important risk factors for rash, whereas male sex was an important risk factor for herpes zoster in the JADER database. The identification of risk factors for skin disorders associated with bendamustine treatment provides useful information for patient management. However, this study has some limitations. The adverse drug cases in the JADER database were reported voluntarily. Thus, spontaneous reporting systems such as the JADER are subject to overreporting, under-reporting, missing data, lack of a denominator, and the presence of confounding factors (e.g., concomitant medications and comorbidities) (29). Therefore, the true incidence of skin disorders cannot be calculated. Although the JADER dataset has more clinical details than other spontaneous reporting system databases such as the FDA Adverse Events Reporting System, further studies are needed to address these limitations. However, reports in spontaneous reporting system databases reflect real-life scenarios and provide a rough indication of signal strength that can be used as a general hypothesis to search for potential ADEs $(30,31)$.

\section{CONCLUSION}

Our analysis of skin disorders related to bendamustine use reported in the spontaneous reporting system databases showed that the association of rash with bendamustine use was affected by sex (female) and age (younger than 70 years). Additionally, the association of herpes zoster with bendamustine was affected by sex (male). Bendamustine is an outpatient chemotherapy regimen, and so we recommend close monitoring of 
female patients or those younger than 70 years who experience rash-like symptoms and male patients who experience herpes zoster-like symptoms.

\section{ACKNOWLEDGEMENTS}

This study was supported by a Grant-in-Aid for Young Scientists (KAKENHI) from the Ministry of Education, Culture, Sports, Science and Technology of Japan (grant number JP19K16429). The authors are grateful to Tadashi Hirooka (TAIHO PHARMA Corporation) for his lecture on Hirooka methods (19) using the JADER database.

\section{CONFLICTS OF INTEREST}

Authors declare no conflict of interest.

\section{REFERENCES}

1. Bogeljić Patekar M, Milunović V, Mišura Jakobac K, Perica D, Mandac Rogulj I, Kursar M, et al. Bendamustine: an old drug in the new era for patients with non-hodgkin lymphomas and chronic lymphocytic leukemia. Acta Clinica Croatica. 2018;57(3):542-553. doi: 10.20471/acc.2018.57.03.18.

2. Knauf WU, Lissichkov T, Aldaoud A, Liberati A, Loscertales J, Herbrecht R, et al. Phase III randomized study of bendamustine compared with chlorambucil in previously untreated patients with chronic lymphocytic leukemia. Journal of clinical oncology: official journal of the American Society of Clinical Oncology. 2009;27(26):4378-4384. doi: 10.1200/JCO.2008.20.8389.

3. Beck J, Schwarzer A, Gläser D, Mügge L-O, Uhlig $\mathrm{J}$, Heyn S, et al. Lenalidomide in combination with bendamustine and prednisolone in relapsed/refractory multiple myeloma: results of a phase 2 clinical trial (OSHO-\#077). Journal of cancer research and clinical oncology. 2017;143(12):2545-2553. doi: 10.1007/s00432017-2504-5.

4. Knauf W, Dingeldein G, Schlag R, Welslau M, Moehler T, Terzer T, et al. First-line therapy with Bendamustine/Prednisone/Bortezomib (BPV) - A GMMG trial for non-transplant eligible symptomatic multiple myeloma patients. European journal of haematology. 2020:10.1111/ejh.13409. doi: 10.1111/ejh.13409.

5. Friedberg JW, Cohen P, Chen L, Robinson KS, Forero-Torres A, La Casce AS, et al. Bendamustine in patients with rituximab-refractory indolent and transformed non-Hodgkin's lymphoma: results from a phase II multicenter, single-agent study.
Journal of Clinical Oncology. 2008;26(2):204-210.

6. Ohmachi K, Ando K, Ogura M, Uchida T, Itoh K, Kubota N, et al. Multicenter phase II study of bendamustine for relapsed or refractory indolent Bcell non-Hodgkin lymphoma and mantle cell lymphoma. Cancer Sci. 2010;101(9):2059-2064. doi: 10.1111/j.1349-7006.2010.01635.x.

7. Belum V, Benhuri B, Postow M, Hellmann M, Lesokhin A, Segal N, et al. Characterisation and management of dermatologic adverse events to agents targeting the PD-1 receptor. European journal of cancer. 2016;60:12-25.

8. Melosky B, Anderson H, Burkes RL, Chu Q, Hao D, Ho V, et al. Pan Canadian Rash trial: a randomized phase III trial evaluating the impact of a prophylactic skin treatment regimen on epidermal growth factor receptor-tyrosine kinase inhibitor-induced skin toxicities in patients with metastatic lung cancer. Journal of Clinical Oncology. 2016;34(8):810-815.

9. Nishikori M, Kitano T, Kobayashi M, Hishizawa M, Kitawaki T, Kondo T, et al. Increased number of peripheral CD8 $+\mathrm{T}$ cells but not eosinophils is associated with late-onset skin reactions caused by bendamustine. International journal of hematology. 2015;102(1):53-58.

10. Inácio $\mathrm{P}$, Cavaco A, Airaksinen $\mathrm{M}$. The value of patient reporting to the pharmacovigilance system: a systematic review. $\mathrm{Br} \mathrm{J}$ Clin Pharmacol. 2017;83(2):227-246. doi: 10.1111/bcp.13098.

11. Lopes P, Nunes T, Campos D, Furlong LI, BauerMehren A, Sanz F, et al. Gathering and exploring scientific knowledge in pharmacovigilance. PLoS One. 2013;8(12):e83016. doi: 10.1371/journal.pone.0083016.

12. Silverman SL. From randomized controlled trials to observational studies. Am J Med. 2009;122(2):114-120. doi: 10.1016/j.amjmed.2008.09.030.

13. Alves C, Macedo AF, Marques FB. Sources of information used by regulatory agencies on the generation of drug safety alerts. Eur J Clin Pharmacol. 2013;69(12):2083-2094. doi: 10.1007/s00228-013-1564-y.

14. Pal SN, Duncombe C, Falzon D, Olsson S. WHO strategy for collecting safety data in public health programmes: complementing spontaneous reporting systems. Drug Saf. 2013;36(2):75-81. doi: 10.1007/s40264-012-0014-6.

15. Uchida M, Kondo Y, Suzuki S, Hosohata K. Evaluation of Acute Kidney Injury Associated With Anticancer Drugs Used in Gastric Cancer in the Japanese Adverse Drug Event Report Database. Ann Pharmacother. 2019;53(12):1200-1206. doi: $10.1177 / 1060028019865870$. 
16. Suga Y, Uchida M, Suzuki S, Sugawara H, Torigoe K, Futamura A, et al. Current Status of Adverse Events Related with Opioid Analgesics in Japan: Assessment Based on Japanese Adverse Drug Event Report Database. Biol Pharm Bull. 2019;42(5):801-806. doi: 10.1248/bpb.b18-00997.

17. Sugawara H, Uchida M, Suzuki S, Suga Y, Uesawa Y, Nakagawa T, et al. Analyses of Respiratory Depression Associated with Opioids in Cancer Patients Based on the Japanese Adverse Drug Event Report Database. Biol Pharm Bull. 2019;42(7):1185-1191. doi: 10.1248/bpb.b1900105.

18. Vijayakumar S, Viswanathan S, Aghoram R. Idiopathic CD4 Lymphocytopenia: Current Insights. ImmunoTargets and Therapy. 2020;9:7993.

19. Hirooka T, Yamada M. Evaluation of risk of adverse reaction using PMDA 'Adverse Drug Reaction Database., Database SAS User Groups Ronbun-syu, 2012.

20. Noguchi Y, Katsuno H, Ueno A, Otsubo M, Yoshida A, Kanematsu Y, et al. Signals of gastroesophageal reflux disease caused by incretinbased drugs: a disproportionality analysis using the Japanese adverse drug event report database. J Pharm Health Care Sci. 2018;4:15. doi: 10.1186/s40780-018-0109-z.

21. Hirschberg E, Gellhorn A, Gump WS. Laboratory evaluation of a new nitrogen mustard, 2-[di-(2chloroethyl)amino-methyl]benzimidazole, and of other 2-chloroethyl compounds. Cancer Res. 1957;17(9):904-910.

22. Darwish M, Bond M, Hellriegel E, Robertson P, Jr., Chovan JP. Pharmacokinetic and pharmacodynamic profile of bendamustine and its metabolites. Cancer Chemother Pharmacol. 2015;75(6):1143-1154. doi: 10.1007/s00280-0152727-6.

23. Mani R, Monteleone C, Schalock PC, Truong T, Zhang XB, Wagner ML. Rashes and other hypersensitivity reactions associated with antiepileptic drugs: A review of current literature. Seizure. 2019;71:270-278. doi: 10.1016/j.seizure.2019.07.015.

24. Fowler T, Bansal AS, Lozsadi D. Risks and management of antiepileptic drug induced skin reactions in the adult out-patient setting. Seizure. 2019;72:61-70. doi: 10.1016/j.seizure.2019.07.003.

25. Knowles SR, Shapiro LE, Shear NH. Anticonvulsant hypersensitivity syndrome: incidence, prevention and management. Drug Saf. 1999;21(6):489-501. doi: 10.2165/00002018199921060-00005.

26. Ogura M, Ishizawa K, Maruyama D, Uike N, Ando $\mathrm{K}$, Izutsu $\mathrm{K}$, et al. Bendamustine plus rituximab for previously untreated patients with indolent B-cell non-Hodgkin lymphoma or mantle cell lymphoma: a multicenter Phase II clinical trial in Japan. Int J Hematol. 2017;105(4):470-477. doi: 10.1007/s12185-016-2146-4.

27. Ahmad DS, Esmadi M, Steinmann WC. Idiopathic CD4 Lymphocytopenia: Spectrum of opportunistic infections, malignancies, and autoimmune diseases. Avicenna J Med. 2013;3(2):37-47. doi: 10.4103/2231-0770.114121.

28. Cencini E, Sicuranza A, Fabbri A, Ferrigno I, Rigacci L, Cox MC, et al. Study of gene polymorphisms as predictors of treatment efficacy and toxicity in patients with indolent non-hodgkin lymphomas and mantle cell lymphoma receiving bendamustine and rituximab. $\mathrm{Br} \mathrm{J}$ Haematol. 2019;184(2):223-231. doi: 10.1111/bjh.15582.

29. van Puijenbroek EP, Bate A, Leufkens HG, Lindquist M, Orre R, Egberts AC. A comparison of measures of disproportionality for signal detection in spontaneous reporting systems for adverse drug reactions. Pharmacoepidemiol Drug Saf. 2002;11(1):3-10. doi: 10.1002/pds.668.

30. Montastruc JL, Sommet A, Bagheri H, LapeyreMestre M. Benefits and strengths of the disproportionality analysis for identification of adverse drug reactions in a pharmacovigilance database. Br J Clin Pharmacol. 2011;72(6):905908. doi: 10.1111/j.1365-2125.2011.04037.x.

31. Pariente A, Gregoire F, Fourrier-Reglat A, Haramburu F, Moore N. Impact of safety alerts on measures of disproportionality in spontaneous reporting databases: the notoriety bias. Drug Saf. 2007;30(10):891-898. doi: 10.2165/00002018200730100-00007 